\title{
Optical trapping of the low index of refraction particles by focused vortex beams and two face-to-face focused beams
}

\author{
MeILING DUAN ${ }^{1 *}$, Hanghang ZHANG ${ }^{2,3}$, JiNHONG LI $^{2}$ \\ ${ }^{1}$ Department of Physics, North University of China, Taiyuan 030051, China \\ ${ }^{2}$ Department of Physics, Taiyuan University of Science and Technology, Taiyuan 030024, China \\ ${ }^{3}$ School of Physics and Optoelectronic Engineering, Xi'dian University, Xi'an 710071, China \\ *Corresponding author: meilingduan@nuc.edu.cn
}

\begin{abstract}
Using the extended Huygens-Fresnel principle and Rayleigh scattering theory, optical trapping of the low index of refraction particles using a focused Gaussian Schell-model (GSM) non-vortex beam, a focused GSM vortex beam, and two face-to-face focused GSM vortex beams have been studied. The results demonstrate that the focused GSM non-vortex beam cannot capture the low index of refraction particles, however, the focused GSM vortex beam can be a two-dimensional trap of particles in the $z$-axis, and the transverse gradient force $F_{\text {grad, } x}$ and the trapping equilibrium region increase as the topological charge $m$ increases. As the focal length $f$ or the refractive index of particles $n_{\mathrm{p}}$ decreases, the radiation forces increase and the trapping ability also enhances. To trap the low index particles in three-dimensional space, we adopt that the two face-to-face focused GSM vortex beams can be used to construct an optical potential well. The transverse gradient force of two face-to-face focused GSM vortex beams is twice that of a single GSM vortex beam. The limit of the radius for the low index of refraction particles that were stably captured has also been determined. The obtained results provide valuable information for trapping and manipulating the low index of refraction particles using GSM vortex beams, which may be applied in micromanipulation, biotechnology, nanotechnology and so on.
\end{abstract}

Keywords: optical trapping, GSM vortex beams, two face-to-face focused beams, radiation forces.

\section{Introduction}

Since AsHKIN's first observation of acceleration and trapping of particles by radiation forces from continuous-wave visible lasers in 1970 [1], optical trapping and manipulation have developed into an important tool for the investigation of the neutral atoms [ $\underline{2}-\underline{4}]$, nanoparticles [ $\underline{5}]$, biological cells [ $\underline{6}-\underline{8}]$, DNA molecules [ $\underline{9}, \underline{10}]$, aerosols [ $[11]$ and other micro-sized dielectric particles $[12,13]$. It is well known that light has momentum and energy, and light radiation force is produced by the exchange of energy and momentum between photons and particles. Optical traps have unique advantages 
since they operate in a non-contact mode and are non-invasive [14-16]. Previously, most optical traps and manipulation have used a focused fundamental Gaussian beam. However, theoretical and experimental studies have demonstrated that the performance of optical traps can be substantially improved and new optical micromanipulation techniques can be realized by exploiting the properties of special classes of laser beams. GAHAGAN and SWARTZLANDER reported the first observation of the simultaneous confinement of both a low-index particle and a high-index particle within a single-beam optical trap by using a strongly focused laser beam containing an optical vortex. They described the trap stability and explored changes in the trapping efficiency owing to the vortex core size, the relative refractive index, and the numerical aperture of the focusing objective from experiment to theory $[17,18]$. The Bessel beam which has the ability of self-reconstruction has been developed to manipulate particles in multiple axial sites [19]. Recently, FAN et al. numerically investigated the trapping effect of tightly focused radially polarized power-exponent-phase vortex beam at the focal plane [20]. We have analyzed the radiation force of focused partially coherent circular edge dislocation beams and focused partially coherent modified Bessel-Gaussian (MBG) beams exerted on two types of particles, and it has been shown that these particles can be simultaneously stably trapped at different positions [21, 22]. In addition, the radi-

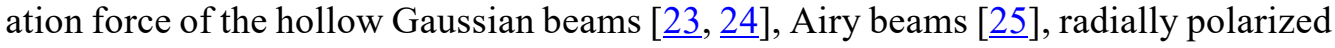
beams [26, 27], and other beams [료] on the microparticles have also been explored. Therefore, optical traps using special laser beams will continue to play an increasingly important role in scientific research in the future. It has been demonstrated that laser beams with Gaussian-like intensity profiles should be used to trap the high index of refraction particles (particles with refractive index $n_{\mathrm{p}}$ greater than that of the ambient value $n_{\mathrm{m}}$, namely, particles with relative refractive index $n_{\mathrm{r}}=n_{\mathrm{p}} / n_{\mathrm{m}}>1$ ), while laser beams with a hollow like intensity profile are applicable for trapping the low index of refraction particles (relative refractive index $n_{\mathrm{r}}<1$ ).

Vortex beams have spiraling wavefronts and orbital angular momentum, which are widely used in applications such as micron-sized particle manipulation, photon counting, optical data storage, optical communications, etc. [며-33]. Significant interest in the propagation characteristics of vortex beams through free space, atmospheric turbulence and biological tissues has been generated in part from the demonstrated theoretical and practical applications of the technique [34-38]. VYAS and SENTHILKUMARAN have proposed the creation of the optical vortex of vortex beams using the modified Michelson interferometer and the modified Mach-Zehnder interferometer [푸, $\underline{40}]$. Roux has described a method for computing the trajectories of optical vortices, and analytically computed the positions of the vortices as a function of the propagation distance $[\underline{41}, \underline{42}$. DIPANKAR et al. have investigated the trajectory of optical vortices in atmospheric turbulence using numerical simulations [43] . We have studied the evolution of coherent vortices and propagation properties of vortex beams through atmospheric turbulence, and found that the beams quality of the vortex beams are less influenced by atmospheric turbulence than those of non-vortex beams [ㄴ4-46]. In our recent paper we have analyzed the classification of coherent vortices creation and the distance of 
topological charge conservation, which showed that the coherent vortices are grouped into three classes according to the creation [구 $\underline{48]}$. Gaussian Schell-model (GSM) vortex beams are a typical example of singularity beams, so we think that this class of beams will prove to be significant in the study of the trapping effect of light.

This paper is devoted to the study of the radiation force on the low index of refraction particles produced by focused GSM non-vortex beams, focused GSM vortex beams, and two face-to-face focused GSM vortex beams in a Rayleigh scattering regime. In Section 2, the analytical expression for the intensity of GSM vortex beams in an optical system is derived. The radiation forces on the low index of refraction particles produced by focused GSM (non-vortex) beams are analyzed in Section 3. The dependence of radiation forces on the topological charge $m$, focal length $f$, the refractive index of particles $n_{\mathrm{p}}$ and particle radius $a$ are analyzed and illustrated for focused GSM vortex beams using numerical examples in Section 4. The scheme and radiation forces of the two face-to-face focused GSM vortex beams used to construct an optical potential well is introduced in Section 5. Finally, Section 6 is a summary of the concluding remarks.

\section{Theoretical model}

The initial field distribution of a Laguerre-Gaussian (LG) beam at the input plane $z=0$ can be expressed as follows $[\underline{32}, \underline{49}, \underline{50}]$ :

$$
E(\mathbf{s}, z=0)=E_{0}\left(\frac{\sqrt{2} r}{w_{0}}\right)^{m} L_{n}^{m}\left(\frac{2 r^{2}}{w_{0}^{2}}\right) \exp \left(-\frac{r^{2}}{w_{0}^{2}}\right) \exp (i m \theta)
$$

where $\mathbf{s}=(r, \theta), r$ and $\theta$ are the radial and azimuthal coordinates, respectively; $E_{0}$ is a positive constant, $w_{0}$ is the waist width, and $m$ and $n$ are the mode orders of the Laguerre polynomial $L_{n}^{m}$. For $m=0$ and $n=0$, Eq. (1) reduces to the initial field of a fundamental Gaussian beam and for $m \neq 0$ and $n=0$, Eq. (1) degenerates to the initial field distribution of a vortex beam [21] .

Using the relations between the Laguerre polynomial and Hermite polynomial [1], we have:

$$
\exp (i m \theta) \mathbf{s}^{m} L_{n}^{m}\left(\mathbf{s}^{2}\right)=\frac{(-1)^{n}}{2^{2 n+m} n !} \sum_{t=0}^{n} \sum_{r=0}^{m} i^{r}\left(\begin{array}{c}
n \\
t
\end{array}\right)\left(\begin{array}{c}
m \\
r
\end{array}\right) H_{2 t+m-r}\left(s_{x}\right) H_{2 n-2 t+r}\left(s_{y}\right)
$$

with $H_{t}$ is the Hermite polynomial of order $t,\left(\begin{array}{l}n \\ t\end{array}\right)\left(\begin{array}{c}m \\ r\end{array}\right)$ represent binomial coefficients. The initial field distribution of vortex beams can be expressed in the following alternative form in Cartesian coordinates:

$$
E(\mathbf{s}, z=0)=E_{0} \frac{1}{2^{m}} \sum_{r=0}^{m} i^{r}\left(\begin{array}{c}
m \\
r
\end{array}\right) H_{2 t+m-r}\left(\frac{\sqrt{2} s_{x}}{w_{0}}\right) H_{-2 t+r}\left(\frac{\sqrt{2} s_{y}}{w_{0}}\right) \exp \left(-\frac{\mathbf{s}^{2}}{w_{0}^{2}}\right)
$$


Introducing the Schell model correlator [ $\underline{52}$ ], the cross-spectral density function of GSM vortex beams at the input plane $z=0$ is expressed as

$$
\begin{aligned}
W_{0}\left(\mathbf{s}_{1}, \mathbf{s}_{2}, 0\right) & =\left\langle E^{*}\left(\mathbf{s}_{1}, 0\right) \bullet E\left(\mathbf{s}_{2}, 0\right)\right\rangle \\
= & \frac{1}{2^{2 m}} E_{0}^{2} \sum_{r_{1}=0}^{m} \sum_{r_{2}=0}^{m}(-i)^{r_{1}} i^{r_{2}}\left(\begin{array}{c}
m \\
r_{1}
\end{array}\right)\left(\begin{array}{c}
m \\
r_{2}
\end{array}\right) H_{m-r_{1}}\left(\frac{\sqrt{2} s_{1 x}}{w_{0}}\right) H_{m-r_{2}}\left(\frac{\sqrt{2} s_{2 x}}{w_{0}}\right) \\
& \times H_{r_{1}}\left(\frac{\sqrt{2} s_{1 y}}{w_{0}}\right) H_{r_{2}}\left(\frac{\sqrt{2} s_{2 y}}{w_{0}}\right) \exp \left(-\frac{\mathbf{s}_{1}^{2}+\mathbf{s}_{2}^{2}}{w_{0}^{2}}\right) \exp \left(-\frac{\left(s_{1}-s_{2}\right)^{2}}{2 \sigma_{0}^{2}}\right)
\end{aligned}
$$

where $\sigma_{0}$ is the spatial correlation length, $E_{0}^{2}$ is related to the input power $P$ and the waist width $w_{0}$ by $E_{0}^{2}=2 P /\left(\pi w_{0}^{2}\right)$.

According to the extended Huygens-Fresnel principle [53], under the paraxial approximation, the cross-spectral density function of GSM vortex beams in the $A B C D$ optical system can be expressed as:

$$
\begin{aligned}
& W\left(\boldsymbol{\rho}_{1}, \boldsymbol{\rho}_{2}, z\right)=\left(\frac{k}{2 \pi B}\right)^{2} \iiint \int W_{0}\left(\mathbf{s}_{1}, \mathbf{s}_{2}, 0\right) \exp \left[-\frac{i k D}{2 z}\left(\boldsymbol{\rho}_{1}^{2}-\boldsymbol{\rho}_{2}^{2}\right)\right] \\
& \times \exp \left\{-\frac{i k}{2 B}\left[A\left(\mathbf{s}_{1}^{2}-\mathbf{s}_{2}^{2}\right)-2\left(\rho_{1 x} s_{1 x}+\rho_{1 y} s_{1 y}-\rho_{2 x} s_{2 x}-\rho_{2 y} s_{2 y}\right)\right]\right\} \mathrm{d} s_{1 x} \mathrm{~d} s_{1 y} \mathrm{~d} s_{2 x} \mathrm{~d} s_{2 y}
\end{aligned}
$$

where $\boldsymbol{\rho}_{1}=\left(\rho_{1 x}, \rho_{1 y}\right)$ and $\boldsymbol{\rho}_{2}=\left(\rho_{2 x}, \rho_{2 y}\right)$ denote the position vector at the $z$ plane, $k$ is the wave number related to the wavelength $\lambda_{0}$ of the input laser by $k=2 \pi / \lambda_{0}, A, B$, and $D$ are the transfer matrix elements of the optical system.

Substituting from Eq. (4) into Eq. (5), we can obtain the cross-spectral density function of the GSM vortex beams through an $A B C D$ optical system by the integral operation as follows:

$$
W\left(\boldsymbol{\rho}_{1}, \boldsymbol{\rho}_{2}, z\right)=E_{0}^{2}\left(\frac{k}{2 \pi B}\right)^{2} 2^{-2 m} \exp \left[-\frac{i k D}{2 z}\left(\boldsymbol{\rho}_{1}^{2}-\boldsymbol{\rho}_{2}^{2}\right)\right] \sum_{r_{1}=0}^{m} \sum_{r_{2}=0}^{m}(-i)^{r_{1}} i^{r_{2}}\left(\begin{array}{l}
m \\
r_{1}
\end{array}\right)\left(\begin{array}{l}
m \\
r_{2}
\end{array}\right) Q_{1} Q_{2}
$$

where the expressions of $Q_{1}$ and $Q_{2}$ have been presented in Appendix.

Using the relation between the intensity and the cross-spectral density at any point of the output plane $I(\boldsymbol{\rho}, z)=W(\boldsymbol{\rho}, \boldsymbol{\rho}, z)$, letting $\rho_{1 x}=\rho_{2 x}=\rho_{x}, \rho_{1 y}=\rho_{2 y}=\rho_{y}$ in Eq. (6), the intensity of the GSM vortex beams at the $z$ plane is written as:

$$
I\left(\rho_{x}, \rho_{y}, z\right)=E_{0}^{2}\left(\frac{k}{2 \pi B}\right)^{2} 2^{-2 m} \sum_{r_{1}=0}^{m} \sum_{r_{2}=0}^{m}(-i)^{r_{1}} i^{r_{2}}\left(\begin{array}{l}
m \\
r_{1}
\end{array}\right)\left(\begin{array}{l}
m \\
r_{2}
\end{array}\right) Q_{1} Q_{2}
$$


For $m=0$, Eq. (7) degenerates to the intensity of the GSM (non-vortex) beam through an $A B C D$ optical system.

Now consider the GSM vortex beam passing the thin lens. The transfer matrix for this optical system is given by

$$
\left[\begin{array}{ll}
A & B \\
C & D
\end{array}\right]=\left[\begin{array}{ll}
1 & z \\
0 & 1
\end{array}\right]\left[\begin{array}{ll}
1 & 0 \\
-1 / f & 1
\end{array}\right]=\left[\begin{array}{ll}
-z_{1} / f & z_{1}+f \\
-1 / f & 1
\end{array}\right]
$$

where $z=z_{1}+f$ is the axial distance from the input plane to the output plane, $f$ is the focal length, $z_{1}$ is the axial distance from the focal plane to the output plane, whose value is negative (or positive) when the particle is located on the left (or right) side of the focal plane.

Now we will consider the radiation forces of a focused GSM vortex beam on a Rayleigh dielectric sphere, with a radius $a$ that is sufficiently smaller than the wavelength of light $\lambda$, i.e., $a \ll \lambda$. In this case, the particle could be treated as a point dipole, and the Rayleigh scattering regime can be used to calculate the radiation forces on the particle irradiated by the focused beam. There are two kinds of radiation forces: scattering force and gradient force. According to the Rayleigh scattering theory, the scattering force and gradient force are expressed as follows [15]:

$$
\begin{aligned}
& \mathbf{F}_{\text {scat }}=\mathbf{e}_{z} \frac{128 n_{\mathrm{m}} \pi^{5} a^{6}}{3 c \lambda^{4}}\left(\frac{n_{\mathrm{r}}^{2}-1}{n_{\mathrm{r}}^{2}+2}\right)^{2} I\left(\rho_{x}, \rho_{y}, z\right) \\
& \mathbf{F}_{\text {grad }}=\frac{2 \pi n_{\mathrm{m}} a^{3}}{c}\left(\frac{n_{\mathrm{r}}^{2}-1}{n_{\mathrm{r}}^{2}+2}\right)^{2} \nabla I\left(\rho_{x}, \rho_{y}, z\right)
\end{aligned}
$$

where $\mathbf{e}_{z}$ is a unity vector along the beam propagation direction, $a$ is the radius of the particle, $c$ is the speed of light in a vacuum, $\lambda=\lambda_{0} / n_{\mathrm{m}}$, and $n_{\mathrm{r}}=n_{\mathrm{p}} / n_{\mathrm{m}}$ specifies the relative refractive index, $n_{\mathrm{p}}$ and $n_{\mathrm{m}}$ denote the refractive indices of the particle and ambient medium, respectively. It can be determined that the scattering force $\mathbf{F}_{\text {scat }}$ is along the direction of beam propagation and is proportional to the beam intensity, and $\mathbf{F}_{\text {grad }}$ is along the gradient of the light intensity. It is well known that the gradient force includes a transverse gradient force $F_{\text {grad, } x}\left(F_{\text {grad, } y}\right)$ on the $x-y$ plane and an axial gradient force $F_{\text {grad, } z}$ along the $z$-direction.

\section{Radiation forces of focused GSM beams on the low index of refraction particles}

The radiation forces of focused GSM (non-vortex) beams acting on the low index of refraction particles at the focal plane are plotted in Fig. 1; the calculation parameters are $m=0, \alpha=10 \mathrm{~nm}, n_{\mathrm{p}}=1$ (air bubble), $n_{\mathrm{m}}=1.33$ (water), $w_{0}=0.5 \mathrm{~mm}, f=5 \mathrm{~mm}$, $\lambda_{0}=0.6328 \mu \mathrm{m}, \sigma_{0}=1 \mathrm{~mm}$, and $E_{0}^{2}=2 \mathrm{~V}^{2} / \mu \mathrm{m}^{2}$. Note that the sign of the gradient force denotes the direction of the force: positive (or negative) $F_{\text {grad }, x}$ indicates that the 

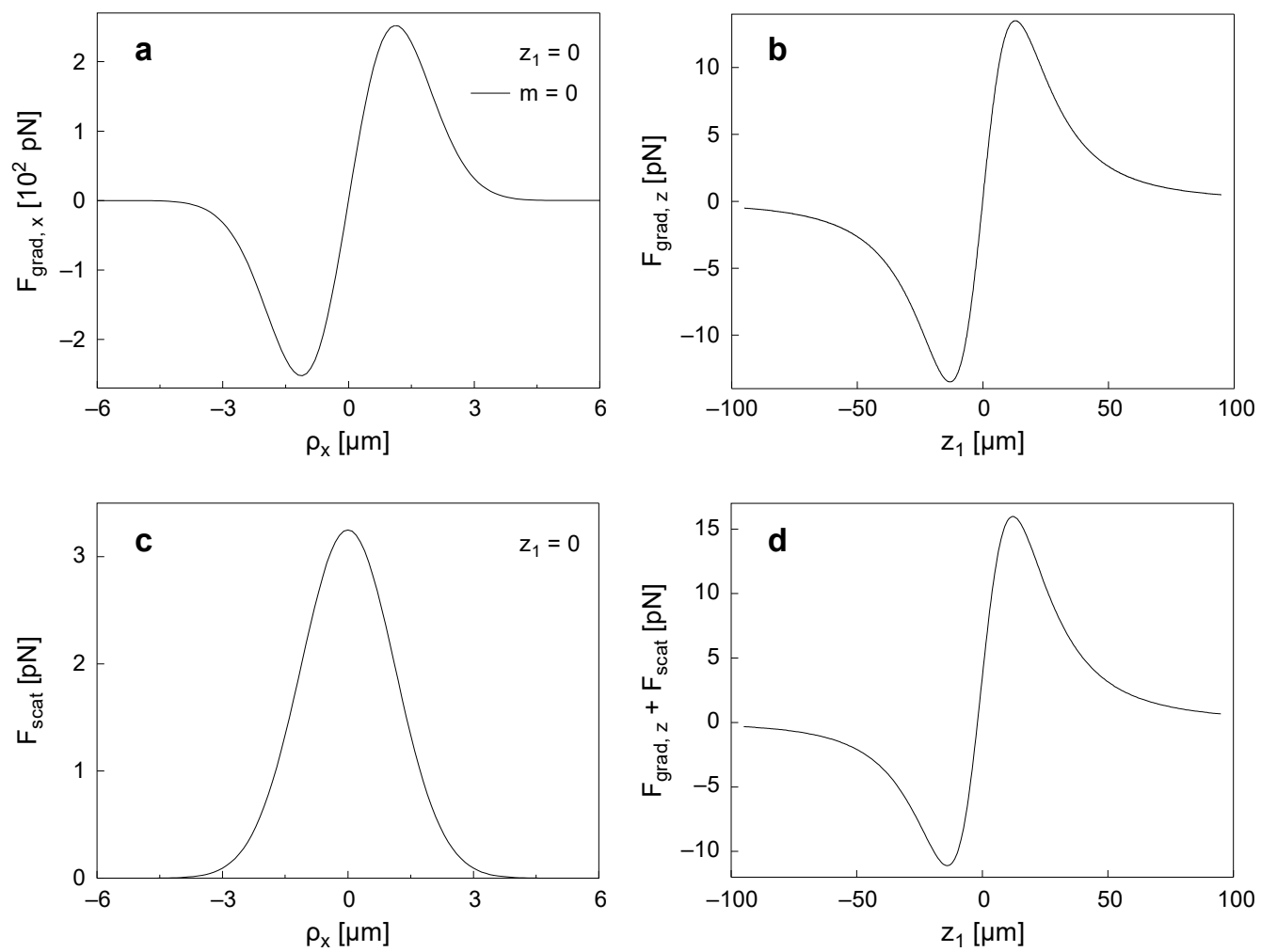

Fig. 1. Radiation forces exerted on the low index of refraction particles by the GSM beam. $F_{\text {grad, } x}$ at the focal plane (a), $F_{\text {grad }, z}(\mathbf{b}), F_{\text {scat }}$ at the focal plane (c), and $F_{\text {grad }, z}+F_{\text {scat }}(\mathbf{d})$.

direction of the transverse gradient force is along the $+x$ (or $-x$ ) direction; for the positive (or negative) $F_{\text {grad, } z}$, this means that the axial gradient force is along the $+z$ (or $-z$ ) direction. From Fig. 1a, we see that the transverse gradient force of the focused GSM beam is negative (or positive) for $\rho_{x}<0$ ( or $\rho_{x}>0$ ), thus it pushes the low index of the refraction particle off the point of $\rho_{x}=0$. From Figs. $1 \mathbf{b}-1 \mathbf{d}$, it is found that the axial gradient force $F_{\text {grad, } z}$ is negative (or positive) for $z_{1}<0$ (or $z_{1}>0$ ), the scattering force $F_{\text {scat }}$ points along the $+z$-axis, and $F_{\text {grad, } z}+F_{\text {scat }}$ can push the low index of the refraction particle off the focus. Therefore, a focused GSM beam cannot trap the low index of refraction particles.

\section{Radiation forces due to focused GSM vortex beams on the low index of refraction particles}

Figure 2 depicts the radiation forces exerted on the low index of refraction particles for different values of the topological charge $m$, the calculation parameters are the same as Fig. 1. From Fig. 2a, it can be determined that the direction of the transverse gradient force $F_{\operatorname{grad}, x}$ is towards the focus $F\left(\rho_{x}=0\right)$ for the low index of refraction particles in 

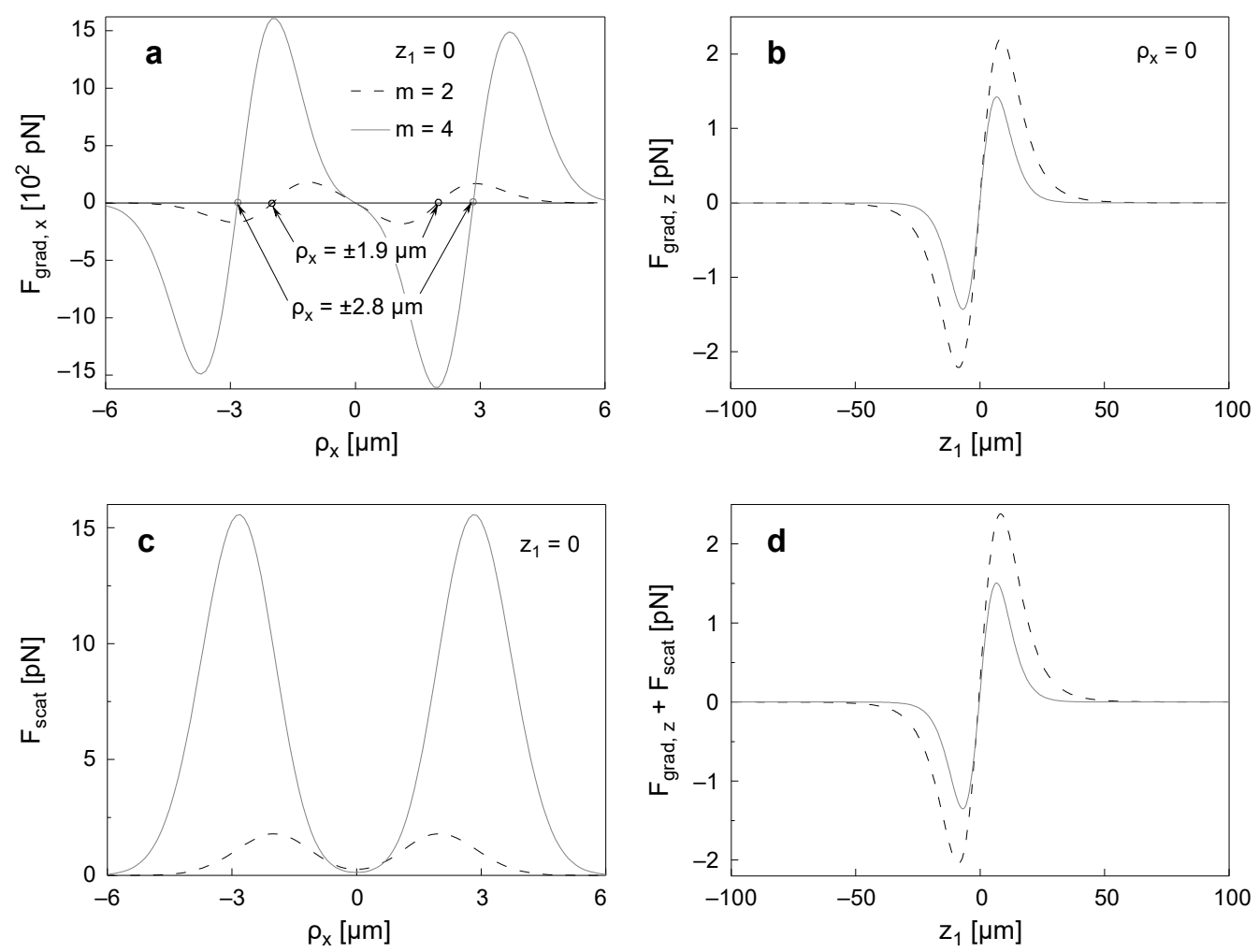

Fig. 2. Radiation forces of focused GSM vortex beams exerted on the low index of refraction particles for different values of topological charge $m ; F_{\text {grad, } x}$ at the focal plane (a), $F_{\text {grad, } z}(\mathbf{b}), F_{\text {scat }}$ at the focal plane (c), and $F_{\text {grad, } z}+F_{\text {scat }}(\mathbf{d})$.

the region $-2.8 \mu \mathrm{m}<\rho_{x}<2.8 \mu \mathrm{m}$ (or $-1.9 \mu \mathrm{m}<\rho_{x}<1.9 \mu \mathrm{m}$ ) for $m=4$ (or $m=2$ ). Thus, the particle is guided to move towards the focus $F$ at the focal plane, i.e., the particle is eventually captured in the focal region (two dimensional). As the topological charge $m$ increases, the transverse gradient force $F_{\text {grad, } x}$ will increase, and the trapping equilibrium region will increase. From Figs. $2 \mathbf{b}-2 \mathbf{d}$, it is evident that the axial gradient force $F_{\text {grad, } z}$ is negative (or positive) for $z_{1}<0$ (or $z_{1}>0$ ), the scattering force $F_{\text {scat }}$ is zero at the focus $\rho_{x}=0$, and the axial radiation force $F_{\text {grad, } z}+F_{\text {scat }}$ can push the low index of refraction particle off the focus. Thus, the use of only a single beam does not allow for the stable trapping of the particle in three dimensions. Therefore, the focused GSM vortex beam only captures low index of refraction particles along the $z$-axis $\left(\rho_{x}=\rho_{y}=0\right)$, and the larger the value of the topological charge $m$, the more easily the particle is stably trapped.

The change of the radiation force of focused GSM vortex beams acting on the low index of refraction particles for different values of the focal length $f$ is represented in Fig. 3 for $m=2$. The other calculation parameters are the same as in Fig. 1. From Fig. 3 it is determined that as the focal length $f$ is increased, the transverse gradient 

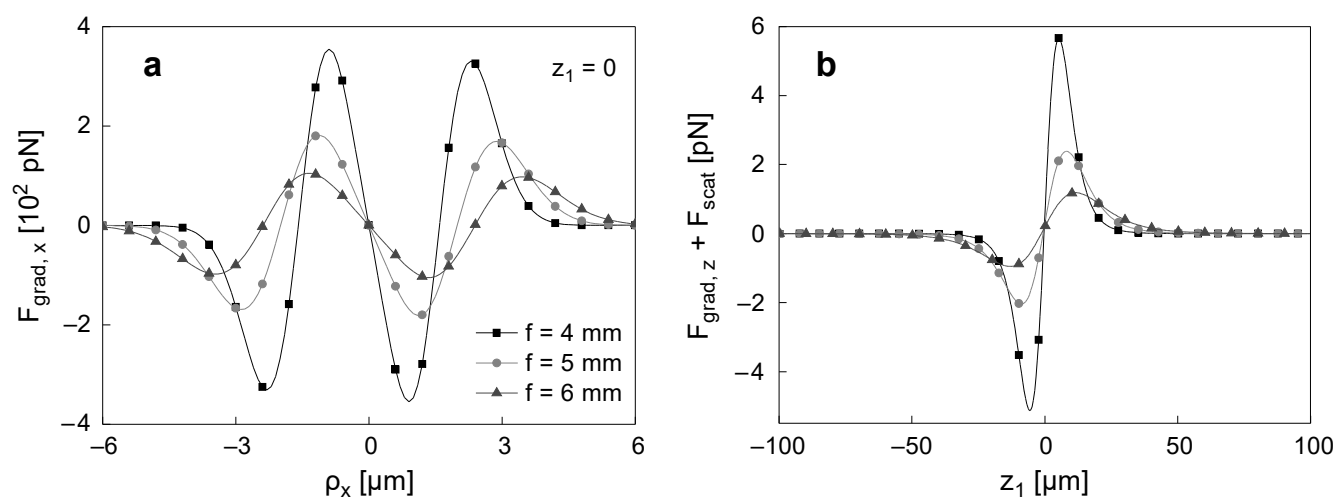

Fig. 3. Radiation forces of focused GSM vortex beams exerted on the low index of refraction particles for different values of focal length $f ; F_{\text {grad, } x}$ at the focal plane (a), and $F_{\text {grad, } z}+F_{\text {scat }}(\mathbf{b})$.
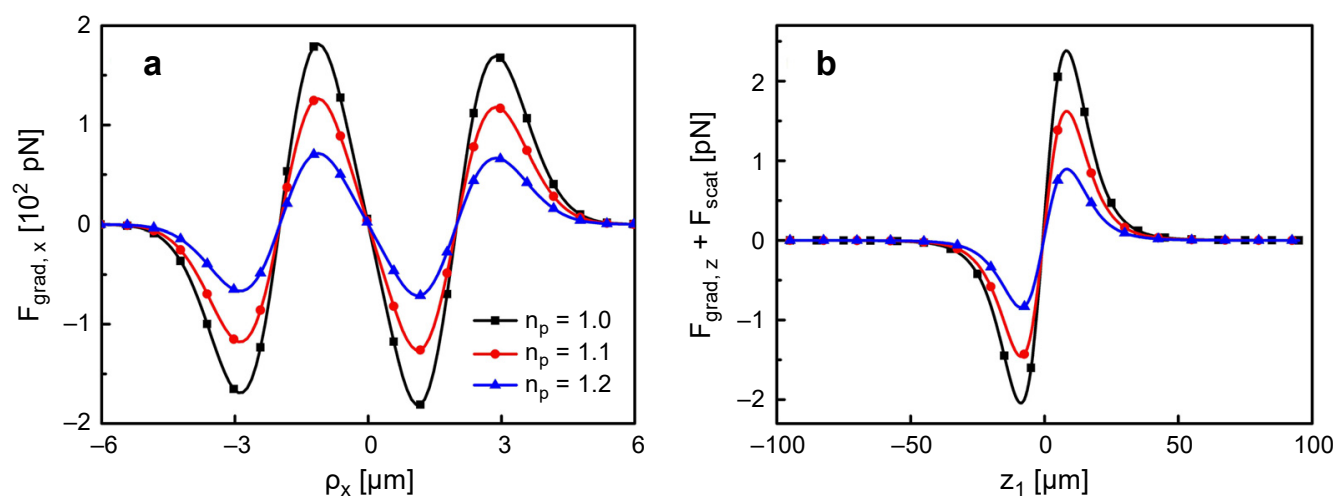

Fig. 4. Radiation forces of focused GSM vortex beams exerted on the low index of refraction particles for different values of refractive index of particles $n_{\mathrm{p}} ; F_{\mathrm{grad}, x}$ at the focal plane (a), and $F_{\text {grad, } z}+F_{\text {scat }}(\mathbf{b})$.

force $F_{\operatorname{grad}, x}$ decreases, the transverse trapping range increases, the axial radiation force $F_{\text {grad, } z}+F_{\text {scat }}$ decreases, and the trapping stability also decreases.

Figure 4 gives the change of the radiation force of focused GSM vortex beams acting on the low index of refraction particles for different values of the refractive index of particles $n_{\mathrm{p}}$ for $m=2$. The other calculation parameters are the same as in Fig. 1. It can be seen that the smaller the refractive index of particles $n_{\mathrm{p}}$ is, the greater the difference between the refractive indices of the ambient and particle $\left(n_{\mathrm{m}}-n_{\mathrm{p}}\right)$ is, i.e., the smaller the relative refractive index $n_{\mathrm{r}}$ is, the greater the transverse gradient force $F_{\text {grad, } x}$ is, and the greater the axial radiation force $F_{\text {grad, } z}+F_{\text {scat }}$ is.

In order to stably trap particles by exploiting the Rayleigh approximation, the following conditions should be satisfied: 1) the axial gradient force must be significantly larger than the scattering force at the position of the maximum axial gradient force, i.e., $R=\left|F_{\text {grad }, z}\right| /\left|F_{\text {scat }}\right| \geq 1$, where $R$ is called the stability criterion. 2) The total force of the particles must overcome the Brownian force $F_{\mathrm{B}}=\left(12 \pi \eta a k_{\mathrm{B}} T\right)^{1 / 2}$ [ㄷ4], where 


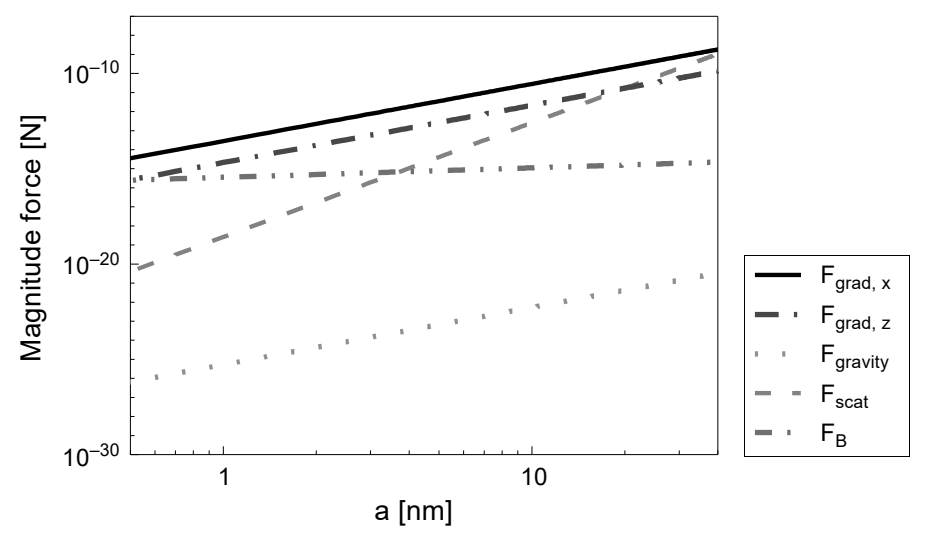

Fig. 5. Comparisons of the variations of $F_{\text {grad }, x}, F_{\text {grad, } z}, F_{\text {scat }}, F_{\mathrm{B}}$ and $F_{\text {gravity }}$ versus the radii of the particles under the condition $m=2$ and $\sigma_{0}=1 \mathrm{~mm}$.

$\eta$ is the viscosity of the ambient medium and $\eta=7.977 \times 10^{-4} \mathrm{~Pa} \cdot \mathrm{s}$ for water at a temperature $T=300 \mathrm{~K}, k_{\mathrm{B}}$ denotes the Boltzmann constant, and $a$ is the radius of the particle. 3) The gradient force must overcome the weight of the particle [ㄷ5]. The magnitude of all the forces versus the radii of the low index of refraction particles is represented in Fig. 5, including the maximum transverse gradient force $F_{\text {grad, } x}$, the maximum axial gradient force $F_{\text {grad, } z}$, the maximum scattering force $F_{\text {scat }}$, the Brownian force $F_{\mathrm{B}}$ and the gravity $F_{\text {gravity }}, m=2$. The other calculation parameters are the same as in Fig. 1 . From Fig. 5, it is determined that as the radius of the particle is increased, the value of $F_{\text {grad, } x}, F_{\text {grad, } z}, F_{\text {scat }}, F_{\mathrm{B}}$ and $F_{\text {gravity }}$ also increase, and the increment of the scattering force is the largest of all these values. When $a>20.2 \mathrm{~nm}$, the scattering force is larger than the axial gradient force. Therefore, the low index of refraction particles with a radius in the range of $0.5 \mathrm{~nm}<a<20.2 \mathrm{~nm}$ can be stably trapped at the focus, i.e., condition (1) is satisfied for $0.5 \mathrm{~nm}<a<20.2 \mathrm{~nm}$. The gradient force can completely overcome the influence of the Brownian force when $a>0.5 \mathrm{~nm}$, so condition (2) is also satisfied for $a>0.5 \mathrm{~nm}$. In this case, the weight of the Rayleigh particle is so small that it can be ignored, so condition (3) is satisfied. Therefore, the focused GSM vortex beam can indeed be used to trap the low index of refraction particles.

\section{Trapping the low index of refraction particles using two face-to-face focused GSM vortex beams}

The aforementioned studies have shown that a single focused GSM vortex beam can capture the low index of refraction particles at the focal plane in only two dimensions, and cannot stably trap particles in three dimensions. To address this problem, we introduce a method for constructing an optical potential well as shown in Fig. 6 by using two face-to-face focused GSM vortex beams. Figure 6 represents the scheme of the two face-to-face focused GSM vortex beams. Two lenses characterized by the same focal length have been placed with their focal planes coinciding. Using the method shown 


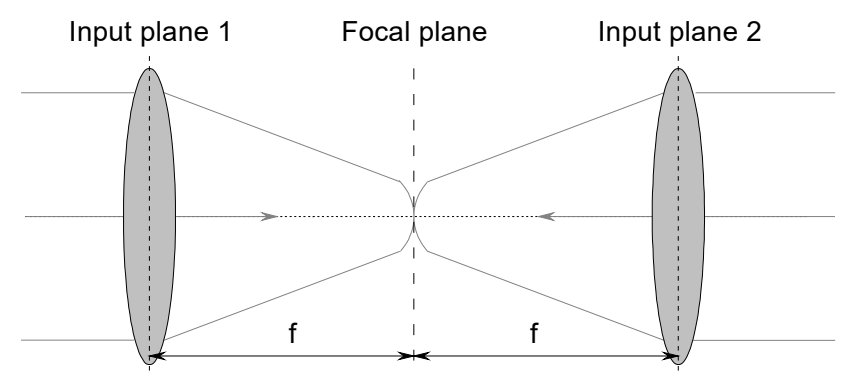

Fig. 6. Scheme of two face-to-face focused GSM vortex beams.

in Fig. 6, the axial radiation forces along the $z$-direction will offset each other at the focal plane. In this case, $R=1$. To check for stability, we can interrupt one GSM vortex beam temporarily, which causes the low index of refraction particles to be accelerated rapidly by the remaining beam along its propagation direction when the other beam is turned on once again, the particle slowly decelerates and returns to its equilibrium region.
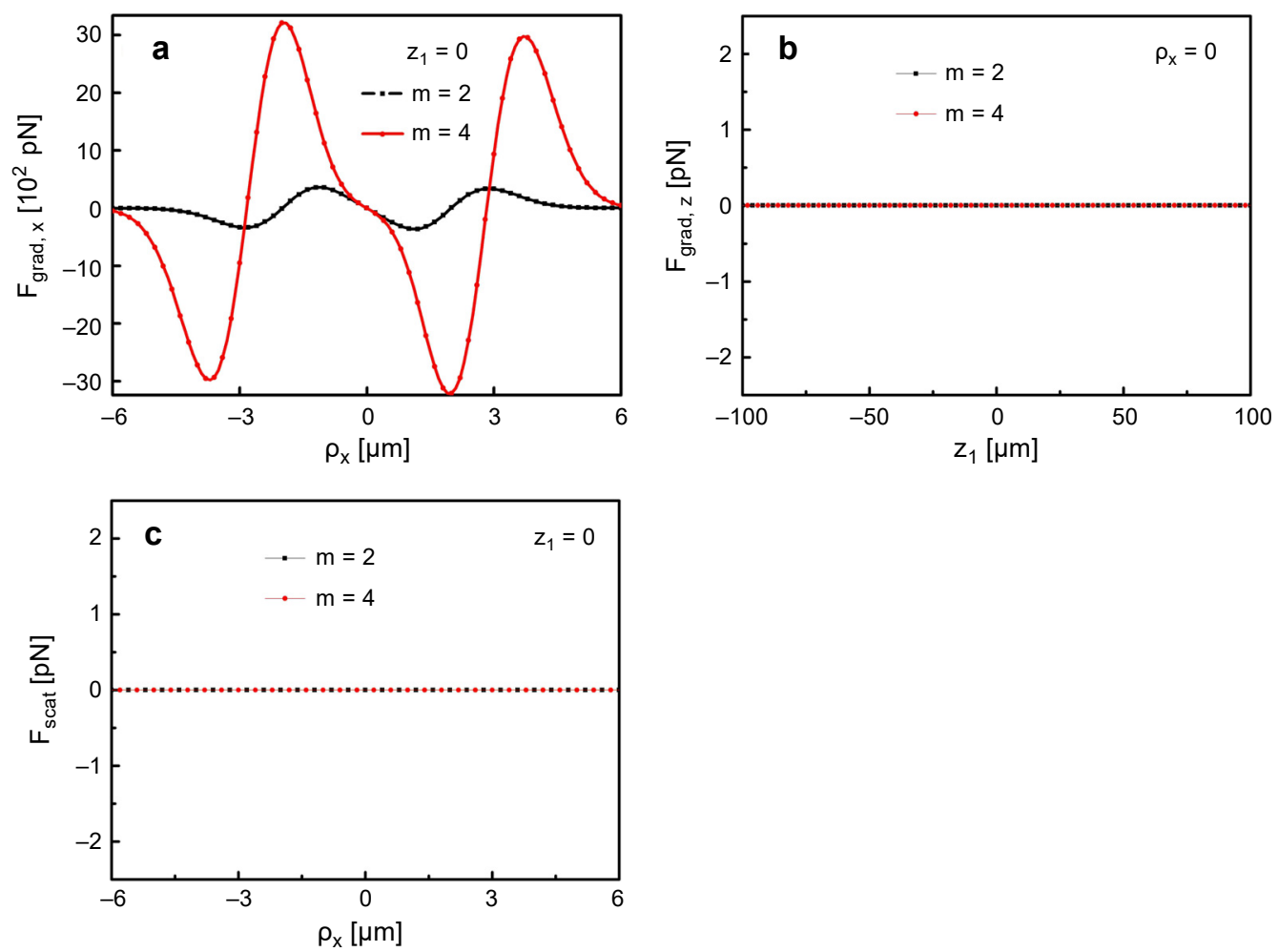

Fig. 7. Radiation forces of two face-to-face focused GSM vortex beams exerted on the low index of refraction particles for different values of the topological charge $m ; F_{\text {grad, } x}$ at the focal plane (a), $F_{\operatorname{grad}, z}(\mathbf{b})$, and $F_{\text {scat }}$ at the focal plane (c). 
Figure 7 depicts the radiation forces exerted on the low index of refraction particles of two face-to-face focused GSM vortex beams, at the calculation parameters the same as used in Fig. 2. Comparing Fig. 7 with Fig. 2, it can be seen that the scattering force and the axial gradient force on the low index of refraction particles of two face-to-face focused GSM vortex beams with the focal plane coinciding will offset each other, the transverse gradient force of two face-to-face focused GSM vortex beams is two times higher than that of a single GSM vortex beam. To sum up, it is indicated that the three -dimensional capture stability can be achieved using two face-to-face focused GSM vortex beams.

\section{Conclusion}

In this paper, optical trapping of the low index of refraction particles using a focused GSM non-vortex beam, GSM vortex beam, and two face-to-face focused GSM vortex beams was investigated based on the extended Huygens-Fresnel principle and Rayleigh scattering theory. The difference between GSM (non-vortex) beams and GSM vortex beams for trapping the low index of refraction particles has been compared. The dependence of the radiation forces on the topological charge $m$, focal length $f$, the refractive index of particles $n_{\mathrm{p}}$ and particle radius $a$ were analyzed and illustrated using numerical examples for focused GSM vortex beams. It has been shown that the focused GSM non-vortex beam cannot capture the low index of refraction particles. The focused GSM vortex beam can trap the low index of refraction particles along the $z$-axis. In this case, as the topological charge $m$ increases, the transverse gradient force $F_{\text {grad, } x}$ and the trapping equilibrium region increase. With an increase of the focal length $f$, the transverse gradient force $F_{\text {grad, } x}$ decreased and the transverse trapping range increases. The radiation force $F_{\text {grad, } x}$ increases with decreasing the refractive index of particles $n_{\mathrm{p}}$. In order to trap the low index of refraction particles in three-dimensional space, we adopted the construction of an optical potential well using two face-to-face focused GSM vortex beams. The transverse gradient force of two face-to-face focused GSM vortex beams is twice that of a single GSM vortex beam. In addition, the range of acceptable radii for the stable capture of the low index of refraction particles was determined. In comparison with the previous work, the focused partially coherent circular edge dislocations beam can be used to trap the high index of refraction particles at the focus $F$, and simultaneously to capture the low index of refraction particles at a dark ring [21], whereas the focused GSM vortex beam can trap the low index of refraction particles to the $z$-axis, thus, the trapping range of the same kind of particles is different obviously for different beams. The results obtained in this paper provide valuable information for trapping and manipulating Rayleigh particles using GSM vortex beams, which may be applied in biotechnology, nanotechnology and other fields. 
(Grant No. 201801D211006), the Fund for Shanxi "1331 Project" key Innovative Research Team (1331KIRT), and the National Natural Science Foundation of China (Grant No. 61601318).

\section{Appendix}

$$
\begin{aligned}
& Q_{1}=\sum_{c_{1}=0}^{\left[\frac{m-r_{1}}{2}\right]} \sum_{d=0}^{m-r_{2}} \sum_{c_{2}=0}^{\left[\frac{d}{2}\right]}\left(\begin{array}{c}
m-r_{2} \\
d
\end{array}\right) \frac{(-1)^{c_{1}+c_{2}}(2 i)^{-\left(m-r_{1}-2 c_{1}+d-2 c_{2}\right)}\left(m-r_{1}\right) ! d !}{c_{1} !\left(m-r_{1}-2 c_{1}\right) ! c_{2} !\left(d-2 c_{2}\right) !} \frac{\pi}{\sqrt{M_{1}}} \\
& \times\left(1-\frac{2}{w_{0}^{2} M_{1}}\right)^{\frac{m-r_{2}}{2}} 2^{-\frac{m-r_{2}}{2}}\left[\frac{2}{w_{0} M_{1} \sigma_{0}^{2}} / \sqrt{1-\frac{2}{w_{0} M_{1}}}\right]^{d-2 c_{2}}\left(\frac{2 \sqrt{2}}{w_{0}}\right)^{m-r_{1}-2 c_{1}} \\
& \times M_{2}^{-\frac{m-r_{1}-2 c_{1}+d-2 c_{2}+1}{2}} \exp \left[\frac{1}{4 M_{1}}\left(\frac{i k \rho_{2 x}}{B}\right)^{2}\right] H_{m-r_{2}-d}\left[\frac{1}{w_{0} M_{1}}\left(-\frac{i k \rho_{2 x}}{B}\right) / \sqrt{1-\frac{2}{w_{0}^{2} M_{1}}}\right]
\end{aligned}
$$

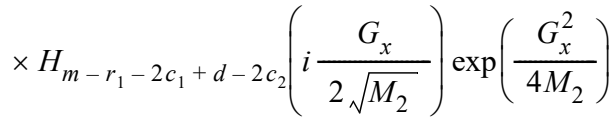

$$
\begin{aligned}
& Q_{2}=\sum_{e_{1}=0}^{\left[\frac{r_{1}}{2}\right]} \sum_{d_{1}=0}^{r_{2}} \sum_{e_{2}=0}^{\left[\frac{d_{1}}{2}\right]}\left(\begin{array}{l}
r_{2} \\
d_{1}
\end{array}\right) \frac{(-1)^{e_{1}+e_{2}}(2 i)^{-\left(r_{1}-2 e_{1}+d_{1}-2 e_{2}\right)} r ! d !}{e_{1} !\left(r_{1}-2 e_{1}\right) ! e_{2} !\left(d_{1}-2 e_{2}\right) !} \frac{\pi}{\sqrt{M_{1}}} \\
& \times\left(1-\frac{2}{w_{0}^{2} M_{1}}\right)^{\frac{r_{2}}{2}} 2^{\frac{r_{2}}{2}}\left[\frac{2}{w_{0} M_{1} \sigma_{0}^{2}} / \sqrt{1-\frac{2}{w_{0} M_{1}}}\right]^{d_{1}-2 e_{2}}\left(\frac{2 \sqrt{2}}{w_{0}}\right)^{r_{1}-2 e_{1}} \\
& \times M_{2}^{-\frac{r_{1}-2 e_{1}+d_{1}-2 e_{2}+1}{2}} \exp \left[\frac{1}{4 M_{1}}\left(\frac{i k \rho_{2 y}}{B}\right)^{2}\right] H_{r_{2}-d_{1}}\left[\frac{1}{w_{0} M_{1}}\left(-\frac{i k \rho_{2 y}}{B}\right) / \sqrt{1-\frac{2}{w_{0}^{2} M_{1}}}\right] \\
& \times H_{r_{1}-2 e_{1}+d_{1}-2 e_{2}}\left(i \frac{G_{y}}{2 \sqrt{M_{2}}}\right) \exp \left(\frac{G_{y}^{2}}{4 M_{2}}\right) \\
& M_{1}=\frac{1}{w_{0}^{2}}+\frac{1}{2 \sigma_{0}^{2}}-\frac{i k A}{2 B} \\
& M_{2}=\frac{1}{w_{0}^{2}}+\frac{1}{2 \sigma_{0}^{2}}+\frac{i k A}{2 B}-\frac{1}{4 M_{1} \sigma_{0}^{4}} \\
& G_{x}=\frac{i k \rho_{1 x}}{B}-\frac{i k \rho_{2 x}}{2 M_{1} B \sigma_{0}^{2}} \\
& G_{y}=\frac{i k \rho_{1 y}}{B}-\frac{i k \rho_{2 y}}{2 M_{1} B \sigma_{0}^{2}}
\end{aligned}
$$




\section{References}

[1] Ashrin A., Acceleration and trapping of particles by radiation pressure, Physical Review Letters 24(4), 1970, pp. 156-159, DOI: 10.1103/PhysRevLett.24.156.

[2] Ashrin A., Trapping of atoms by resonance radiation pressure, Physical Review Letters 40(12), 1978, pp. 729-732, DOI: 10.1103/PhysRevLett.40.729.

[3] Yavuz D.D., Kulatunga P.B., Urban E., Johnson T.A., Proite N., Henage T., Walker T.G., SAFFMAN M., Fast ground state manipulation of neutral atoms in microscopic optical traps, Physical Review Letters 96(6), 2006, article 063001, DOI: 10.1103/PhysRevLett.96.063001.

[4] Zhang P., Li G., Zhang T., Subwavelength optical dipole trap for neutral atoms using a microcapillary tube tip, Journal of Physics B: Atomic, Molecular and Optical Physics 50(4), 2017, article 045005, DOI: 10.1088/1361-6455/aa5518.

[5] Yannopapas V., Paspalakis E., Electrodynamic multiple-scattering method for the simulation of optical trapping atop periodic metamaterials, Journal of Modern Optics 65(13), 2018, pp. 1507-1514, DOI: $10.1080 / 09500340.2018 .1455909$.

[6] Zhong M., Wei X., Zhou J., Wang Z., Li Y., Trapping red blood cells in living animals using optical tweezers, Nature Communications 4(4), 2013, article 1768, DOI: 10.1038/ncomms2786.

[7] Samadi A., Zhang C., Chen J., Reihani S.N.S., Chen Z., Evaluating the toxic effect of an antimicrobial agent on single bacterial cells with optical tweezers, Biomedical Optics Express 6(1), 2015, pp. 112-117, DOI: 10.1364/BOE.6.000112.

[8] Duan M., Zhang C., Li J., Coherence and polarization properties of laser propagating through biological tissues, Journal of Photochemistry and Photobiology B: Biology 172, 2017, pp. 88-94, DOI: $10.1016 /$ j.jphotobiol.2017.05.007.

[9] Tskhovrebova L., Trinick J., Sleep J.A., Simmons R.M., Elasticity and unfolding of single molecules of the giant muscle protein titin, Nature 387(6630), 1997, pp. 308-312, DOI: 10.1038/387308a0.

[10] Wang M.D., Yin H., Landick R., Gelles J., Block S.M., Stretching DNA with optical tweezers, Biophysical Journal 72(3), 1997, pp. 1335-1346, DOI: 10.1016/S0006-3495(97)78780-0.

[11] Zhang P., Zhang Z., Prakash J., Huang S., Hernandez D., Salazar M., Christodoulides D.N., CHEN Z., Trapping and transporting aerosols with a single optical bottle beam generated by moiré techniques, Optics Letters 36(8), 2011, pp. 1491-1493, DOI: 10.1364/OL.36.001491.

[12] Calander N., Willander M., Optical trapping of single fluorescent molecules at the detection spots of nanoprobes, Physical Review Letters 89(14), 2002, article 143603, DOI: 10.1103/PhysRevLett. $\underline{89.143603 .}$.

[13] Gu B., Pan Y., Rui G., Xu D., Zhan Q., Cui Y., Polarization evolution characteristics of focused hybridly polarized vector fields, Applied Physics B 117(3), 2014, pp. 915-926, DOI: 10.1007/ s00340-014-5909-8.

[14] Svoboda K., Block S.M., Optical trapping of metallic Rayleigh particles, Optics Letters 19(13), 1994, pp. 930-932, DOI: 10.1364/OL.19.000930.

[15] Harada Y., AsaKuRa T., Radiation forces on a dielectric sphere in the Rayleigh scattering regime, Optics Communications 124(5-6), 1996, pp. 529-541, DOI: 10.1016/0030-4018(95)00753-9.

[16] Shu J., Chen Z., Pu J., Radiation forces on a Rayleigh particle by highly focused partially coherent and radially polarized vortex beams, Journal of the Optical Society of America A 30(5), 2013, pp. 916-922, DOI: 10.1364/JOSAA.30.000916.

[17] Gahagan K.T., Swartzlander G.A., Trapping of low-index microparticles in an optical vortex, Journal of the Optical Society of America B 15(2), 1998, pp. 524-534, DOI: 10.1364/JOSAB.15.000524.

[18] Gahagan K.T., SwartZlander G.A., Simultaneous trapping of low-index and high-index microparticles observed with an optical-vortex trap, Journal of the Optical Society of America B 16(4), 1999, pp. 533-537, DOI: 10.1364/JOSAB.16.000533.

[19] Garces-Chavez V., McGloin D., Melville H., Sibbett W., Dholakia K., Simultaneous micromanipulation in multiple planes using a self-reconstructing light beam, Nature 419(6903), 2002, pp. 145-147, DOI: 10.1038/nature01007. 
[20] FAN C., LiU Y., WANG X., Chen Z., PU J., Trapping two types of particles by using a tightly focused radially polarized power-exponent-phase vortex beam, Journal of the Optical Society of America A 35(6), 2018, pp. 903-907, DOI: 10.1364/JOSAA.35.000903.

[21] Zhang H., Li J., Cheng K., Duan M., Feng Z., Trapping two types of particles using a focused partially coherent circular edge dislocations beam, Optics \& Laser Technology 97, 2017, pp. 191-197, DOI: $10.1016 /$ j.optlastec.2017.06.025.

[22] Duan M., Zhang H., Li J., Cheng K., Wang G., YAng W., Trapping two types of particles using a focused partially coherent modified Bessel-Gaussian beam, Optics and Lasers in Engineering 110, 2018, pp. 308-314, DOI: 10.1016/j.optlaseng.2018.06.015.

[23] ZhaO C., WANG L., Lu X., Radiation forces on a dielectric sphere produced by highly focused hollow Gaussian beams, Physics Letters A 363(5-6), 2007, pp. 502-506, DOI:10.1016/j.physleta.2006.11.028.

[24] Xu H., Zhang W., Qu J., Huang W., Optical trapping Rayleigh dielectric particles with focused partially coherent dark hollow beams, Journal of Modern Optics 62(21), 2015, pp. 1839-1848, DOI: 10.1080/09500340.2015.1045947.

[25] Jiang Y., CAo Z., Shao H., Zheng W., Zeng B., Lu X., Trapping two types of particles by modified circular Airy beams, Optics Express 24(16), 2016, pp. 18072-18081, DOI: 10.1364/OE.24.018072.

[26] Zhang Y., Ding B., Suyama T., Trapping two types of particles using a double-ring-shaped radially polarized beam, Physical Review A 81(2), 2010, article 023831, DOI: 10.1103/PhysRevA.81.023831.

[27] Zhuang Y., Zhang Y., Ding B., Suyama T., Trapping Rayleigh particles using highly focused higher-order radially polarized beams, Optics Communications 284(7), 2011, pp. 1734-1739, DOI: 10.1016/j.optcom.2010.12.018.

[28] Li M., Yan S., Liang Y., Zhang P., Yao B., Spinning of particles in optical double-vortex beams, Journal of Optics 20(2), 2018, article 025401, DOI: 10.1088/2040-8986/aaa0e9.

[29] Mair A., Vaziri A., Weins G., Zeilinger A., Entanglement of the orbital angular momentum states of photons, Nature 412(6844), 2001, pp. 313-316, DOI: 10.1038/35085529.

[30] Yang Y., Dong Y., Zhao C., Cai Y., Generation and propagation of an anomalous vortex beam, Optics Letters 38(24), 2013, pp. 5418-5421, DOI: 10.1364/OL.38.005418.

[31] Li J., ZENG J., Dynamic evolution of coherent vortex dipole in atmospheric turbulence, Optics Communications 383, 2017, pp. 341-348, DOI: 10.1016/j.optcom.2016.09.031.

[32] Li J., Gao P., Cheng K., Duan M., Dynamic evolution of circular edge dislocations in free space and atmospheric turbulence, Optics Express 25(3), 2017, pp. 2895-2908, DOI: 10.1364/OE.25.002895.

[33] QIU C.-W., YANG Y., Vortex generation reaches a new plateau, Science 357(6352), 2017, p. 645, DOI: $10.1126 /$ science.aan6359.

[34] Yang Y., ThirunavukKarasu G., Babiker M., Yuan J., Orbital-angular-momentum mode selection by rotationally symmetric superposition of chiral states with application to electron vortex beams, Physical Review Letters 119(9), 2017, article 094802, DOI: 10.1103/PhysRevLett.119.094802.

[35] Yang Y., Dong Y., Zhao C., Liu Y., Cai Y., Autocorrelation properties of fully coherent beam with and without orbital angular momentum, Optics Express 22(3), 2014, pp. 2925-2932, DOI: $10.1364 /$ OE.22.002925.

[36] Li J., Wang W., DuAn M., WeI J., Influence of non-Kolmogorov atmospheric turbulence on the beam quality of vortex beams, Optics Express 24(18), 2016, pp. 20413-20423, DOI: 10.1364/OE.24.020413.

[37] Duan M., Wu Y., Su N., Changes of the polarization states of random electromagnetic vortex beams propagating in biological tissues, Optica Applicata 48(2), 2018, pp. 297-309, DOI: 10.5277/oa180212.

[38] Li J., Zhang H., LÜ B., Partially coherent vortex beams propagating through slant atmospheric turbulence and coherence vortex evolution, Optics \& Laser Technology 42(2), 2010, pp. 428-433, DOI: 10.1016/j.optlastec.2009.08.019.

[39] Vyas S., Senthilkumaran P., Interferometric optical vortex array generator, Applied Optics 46(15), 2007, pp. 2893-2898, DOI: 10.1364/AO.46.002893.

[40] Vyas S., Senthilkumaran P., Vortex array generation by interference of spherical waves, Applied Optics 46(32), 2007, pp. 7862-7867, DOI: 10.1364/AO.46.007862. 
[41] Roux F.S., Paraxial modal analysis technique for optical vortex trajectories, Journal of the Optical Society of America B 20(7), 2003, pp. 1575-1580, DOI: 10.1364/JOSAB.20.001575.

[42] Roux F.S., Distribution of angular momentum and vortex morphology in optical beams, Optics Communications 242(1-3), 2004, pp. 45-55, DOI: 10.1016/j.optcom.2004.08.006.

[43] Dipankar A., Marchiano R., Sagaut P., Trajectory of an optical vortex in atmospheric turbulence, Physical Review E 80(4), 2009, article 046609, DOI: 10.1103/PhysRevE.80.046609.

[44] LI J., LÜ B., Propagation of Gaussian Schell-model vortex beams through atmospheric turbulence and evolution of coherent vortices, Journal of Optics A: Pure and Applied Optics 11(4), 2009, article 045710, DOI: 10.1088/1464-4258/11/4/045710.

[45] LI J., LÜ B., Composite coherence vortices in superimposed partially coherent vortex beams and their propagation through atmospheric turbulence, Journal of Optics A: Pure and Applied Optics 11(7), 2009, article 075401, DOI: $10.1088 / 1464-4258 / 11 / 7 / 075401$.

[46] Li J., Zhang H., LÜ B., Composite coherence vortices in a radial beam array propagating through atmospheric turbulence along a slant path, Journal of Optics 12(6), 2010, article 065401, DOI: 10.1088/2040-8978/12/6/065401.

[47] Li J., Zeng J., Duan M., Classification of coherent vortices creation and distance of topological charge conservation in non-Kolmogorov atmospheric turbulence, Optics Express 23(9), 2015, pp. 11556-11565, DOI: 10.1364/OE.23.011556.

[48] Zeng J., Li J., Dynamic evolution and classification of coherent vortices in atmospheric turbulence, Optica Applicata 45(3), 2015, pp. 299-308, DOI: 10.5277/oa150303.

[49] ZAUderer E., Complex argument Hermite-Gaussian and Laguerre-Gaussian beams, Journal of the Optical Society of America A 3(4), 1986, pp. 465-469, DOI: 10.1364/JOSAA.3.000465.

[50] Qu J., Zhong Y., Cui Z., CAi Y., Elegant Laguerre-Gaussian beam in a turbulent atmosphere, Optics Communications 283(14), 2010, pp. 2772-2781, DOI: 10.1016/j.optcom.2010.03.022.

[51] Kimel I., Elias L.R., Relations between Hermite and Laguerre Gaussian modes, IEEE Journal of Quantum Electronics 29(9), 1993, pp. 2562-2567, DOI: 10.1109/3.247715.

[52] Zahid M., Zubairy M.S., Directionality of partially coherent Bessel-Gauss beams, Optics Communications 70(5), 1989, pp. 361-364, DOI: 10.1016/0030-4018(89)90131-4.

[53] Andrews L.C., Phillips R.L., Laser Beam Propagation through Random Media, 2nd Ed., SPIE Press, Bellingham 2005, DOI: 10.1117/3.626196.

[54] Окамото K., Kawata S., Radiation force exerted on subwavelength particles near a nanoaperture, Physical Review Letters 83(22), 1999, pp. 4534-4537, DOI: 10.1103/PhysRevLett.83.4534.

[55] ZhaO C., WANG L., Lu X., Radiation forces of highly focused Bessel-Gaussian beams on a dielectric sphere, Optik 119(10), 2008, pp. 477-480, DOI: 10.1016/j.ijleo.2006.11.013.

Received June 27, 2019

in revised form October 1, 2019 